\title{
A IMPORTÂNCIA DA ASSISTÊNCIA INTEGRAL À SAÚDE DA MULHER NA PREVENÇÃO DO CÂNCER DE MAMA: UM PROJETO DE INTERVENÇÃOO
}

\author{
ASSISTANCE OF IMPORTANCE INTEGRAL HEALTH OF WOMEN IN \\ BREAST CANCER PREVENTION: A POLICY PROJECT \\ Fernanda de Oliveira Souza ${ }^{1}$, Elaine Andrade Leal Silva², Aline Magalhães Bessa \\ Andrade $^{3}$, Tamille Marins Santos Cerqueira ${ }^{4}$ \\ ${ }^{1}$ Universidade Estadual de Feira de Santana/Saúde/Programa de Pós Graduação em Saúde Coletiva, \\ nandaolisouza@gmail.com \\ ${ }^{2}$ Universidade Federal do Recôncavo da Bahia/Saúde/UFRB, ealealsilva@ hotmail.com \\ ${ }^{3}$ Universidade Federal da Bahia/Enfermagem/UFBA, linebmagalhaes@ hotmail.com \\ ${ }^{4}$ Universidade Federal do Recôncavo da Bahia/Enfermagem/UFRB, tamillemarins@ @otmail.com
}

\section{RESUMO}

A problemática do câncer no Brasil é relevante pelo impacto no perfil epidemiológico que essa doença vem apresentando, e, com isso, o tema tem conquistado espaço prioritário nas agendas políticas do governo. O objetivo deste trabalho foi descrever um relato de experiência do desenvolvimento do projeto "Amigas da Mama". Tratou-se de um relato de uma experiência resultante de ações de extensão universitária de discentes e docente de enfermagem da Universidade Federal do Recôncavo da Bahia, além de integrantes da equipe de saúde da família. A população alvo foi todas as mulheres cadastradas em uma das Unidades de Saúde da Família (USF) de um município do recôncavo baiano. Foram realizadas atividades de educação em saúde nas salas de espera de atendimento; educação em serviço para equipe de técnicos em enfermagem e agentes comunitários de saúde; confecção de um fluxograma para direcionar a dispensação das mamografias e o encerramento do projeto com o dia de prevenção e combate ao câncer de mama. $\mathrm{O}$ presente projeto proporcionou a vivência da troca de saberes entre acadêmicos, equipe de saúde da família e comunidade, ressignificando o processo de educação, baseado na construção compartilhada de conhecimento.

Palavras-chave: Câncer de mama. Extensão. Educação em saúde.

\begin{abstract}
The cancer problem in Brazil is relevant for the impact on the epidemiological profile that this disease has shown, and with it, the topic has gained priority space in government policy agendas. The objective of this study was to describe an experience report of project development "Friends of Mama." It was an account of an experience resulting from university extension actions of students and faculty of nursing at the Federal University of Bahia Reconcavo, and family health team members. The target population was all women registered in one of the Family Health Units in a city of Bahia Recôncavo. Education activities were carried out in health in the waiting rooms of service; service education for technical staff in nursing and community health workers; making a flowchart to guide the dispensation of mammograms and project closure with the day to prevent and fight against breast cancer. This project provided the experience of exchange of knowledge between academics, health team of family and community, giving new meaning to the process of education, based on shared knowledge construction.
\end{abstract}

Key words: Breast cancer. Extension. Health education. 


\section{Ensino, Saúde e Ambiente - V9 (1), pp. 33-42, Abril, 2016.}

\section{Introdução}

A mortalidade por câncer nos últimos anos cresce, principalmente em função do crescimento populacional, processo de industrialização e mudanças nos hábitos de vida (MAFRA, 2005). O câncer de mama é provavelmente o mais temido pelas mulheres, devido à sua alta incidência e, sobretudo pelos seus efeitos psicológicos, que afetam a percepção da sexualidade e a própria imagem pessoal (BRASIL, 2006).

A problemática do câncer no Brasil é relevante pelo impacto no perfil epidemiológico que essa doença vem apresentando, e, com isso, o tema tem conquistado espaço prioritário nas agendas políticas do governo. O conhecimento sobre a incidência dessa doença permite a alocação recursos de forma direcionada para a modificação positiva desse cenário na população brasileira (INCA, 2011).

Esta integralidade foi reafirmada com a publicação da Lei no 11.664 , de 29 de abril de 2008, que trata da efetivação de ações de saúde relacionadas à prevenção, detecção e tratamento dos cânceres do colo do útero e de mama. Essa lei assegura que o exame da mama é garantido a todas as mulheres acima de 40 anos de idade e caso a mulher necessite de complementação de diagnóstico, esta deverá ser encaminhada a serviços de maior complexidade (BRASIL, 2010).

Vale ressaltar que quando mais precoce for feito o diagnóstico de câncer maior a probabilidade de cura, entretanto o rastreamento torna-se ainda mais relevante que a detecção precoce, uma vez que este permite a identificação da doença em sua fase préclínica, pois apesar de ser considerado como carcinoma de prognóstico favorável, se diagnosticado e tratado oportunamente, as taxas de mortalidade por neoplasia mamária continuam elevadas no Brasil, muito provavelmente porque a doença ainda seja diagnosticada em estádios avançados (FOGAÇA; GARROTE, 2004).

Desta forma, as estratégias adotadas para o controle do câncer de mama devem estar direcionadas as ações de prevenção, visando à redução dos fatores de risco relacionados ao câncer de mama, detecção precoce (identificar o câncer o mais cedo possível), tratamento do câncer, reabilitação e cuidados paliativos (INCA, 2011).

Destarte a Unidade de Saúde deve estar organizada para realizar o exame clínico das mamas das mulheres, sendo este considerado como parte fundamental da propedêutica para o diagnóstico de câncer, além de constituir a base para a solicitação dos exames complementares, a exemplo da mamografia (BRASIL, 2004). O serviço de saúde deve encaminhar aquelas cujo resultado mamográfico ou cujo exame clínico indiquem necessidade de maior investigação (BRASIL, 2006). 
Ensino, Saúde e Ambiente - V9 (1), pp. 33-42, Abril, 2016.

Para isso torna-se imprescindível a capacitação da equipe de saúde da família visando à sensibilização da população feminina sobre a atenção especial que deve ser dada às mamas. Além disso, deveriam ser incentivadas campanhas sobre a importância da participação de mulheres assintomáticas em programas específicos para doenças das mamas (FOGAÇA; GARROTE, 2004).

O movimento popular internacionalmente conhecido como Outubro Rosa simboliza a luta contra o câncer de mama. Este movimento começou nos Estados Unidos, onde vários Estados tinham ações isoladas referentes ao câncer de mama, posteriormente com a aprovação do Congresso Americano o mês de Outubro se tornou o mês nacional de prevenção do câncer de mama, tendo como foco principal a mobilização social e o desenvolvimento de ações preventivas referentes à doença. (AFECC, 2012). A partir da compreensão da educação em saúde como caminho que visa articular dimensões complementares com vistas à construção de respostas sociais significativas, é possível compreender que a educação não trata de definir comportamentos corretos para os demais, mas de criar oportunidades de reflexão crítica e interação dialógica entre sujeitos sociais (MEYER et al., 2006)

SOUZA e colaboradores (2013) demonstraram a importância de trocar experiências, para compreender melhor o processo da mulher frente ao câncer de mama. O objetivo deste estudo foi descrever um relato de experiência referente ao desenvolvimento do projeto "Amigas da Mama" integrando as ações de ensino, pesquisa, extensão e intervenção na prevenção de Câncer de Mama.

\section{Metodologia}

Este relato de uma experiência é resultante de ações de extensão universitária de discentes de enfermagem em estágio supervisionado I da Universidade Federal do Recôncavo da Bahia.

O mês de outubro é considerado mês de combate ao câncer de mama ou outubro rosa. Tendo em vista a realização de um projeto de intervenção a ser realizado pelas discentes, buscou-se aliar o mês referido: "Outubro Rosa" ao propósito de promover educação em saúde a respeito do câncer de mama para parte das 2.383 mulheres, cadastradas em uma das vinte e três Unidades de Saúde da Família de Santo Antônio de Jesus.

Esta cidade está localizada na região do Recôncavo Sul da Bahia, com população estimada de 101.548 habitantes, o município possui rede de saúde 


\section{Ensino, Saúde e Ambiente - V9 (1), pp. 33-42, Abril, 2016.}

organizada. O projeto foi iniciado no mês de outubro, por discentes do curso de enfermagem e docente da UFRB, pelas profissionais psicóloga, nutricionista e fisioterapeuta integrantes do NASF (Núcleo de Apoio a Saúde da Família) e integrantes da equipe de saúde da família (enfermeira, técnicas de enfermagem e agentes comunitários de saúde). A população alvo foram todas as mulheres cadastradas em uma das Unidades de Saúde da Família do município.

Inicialmente realizou-se o aprofundamento do conhecimento das discentes de enfermagem sobre o Câncer de mama, foi solicitada aos Agentes Comunitários de Saúde (ACS) a relação nominal do quantitativo de mulheres existentes na área de abrangência da unidade, e se possível a identificação de casos de câncer de mama existente na população.

Desta forma, foi discutido juntamente com a equipe o diagnóstico situacional da área, justificando assim, a necessidade de dialogar com a população sobre a temática proposta. Além disso, o quantitativo reduzido de requisições de mamografia e consequentemente a insatisfação da população feminina, suscitaram a discussão sobre o tema.Os dados epidemiológicos mais atualizados, foram utilizados para elaboração/implementação do projeto de intervenção que teve como título: Amigas da Mama.

Em seguida, buscou-se confeccionar um calendário de atividades propostas para o mês de outubro. O projeto deste calendário foi construído pela equipe de trabalho da Estratégia de Saúde da Família, pelo NASF, discentes do curso de enfermagem e docente do componente curricular Estágio Supervisionado I.

O principal recurso utilizado foi o humano, este necessitou fundamentalmente de treinamento. Todos participantes do projeto (técnicos de enfermagem, enfermeira, agentes comunitários, recepcionistas e outros profissionais de nível superior) foram capacitados para realizar o trabalho durante todo o mês e se tornarem multiplicadores/facilitadores dos conhecimentos.

As atividades que foram previstas para o outubro rosa foram: realização de educação em saúde nas salas de espera de atendimento, dentre os mais diversos programas ofertados pela USF; educação em serviço para equipe de técnicos em enfermagem e agentes comunitários de saúde; confecção de um fluxograma que direcionasse a dispensação das mamografias ofertadas pelo município e participação do projeto de intervenção ao final do mês "outubro rosa" no ano de 2012: mês nacional prevenção e combate ao câncer de mama. 
Ensino, Saúde e Ambiente - V9 (1), pp. 33-42, Abril, 2016.

\section{Resultados e Discussão}

\section{Educação em saúde na sala de espera}

Sob uma perspectiva problematizadora da educação popular em saúde, uma das metas do projeto era utilizar a sala de espera da unidade de saúde como um local de educação para a comunidade. Durante todo o mês de outubro foram levantadas diferentes abordagens para tratar sobre o câncer de mama nos mais diversos grupos que estivessem aguardando atendimento para cada programa que a unidade de saúde oferta atendimento. Utilizou-se a agenda diária da profissional enfermeira para construção das educações a serem realizadas, os grupos atingidos foram hipertensos, diabéticos e gestantes.

Para o grupo de hipertensos e diabéticos foi exemplificado que o câncer de mama ocorre principalmente em mulheres com mais de 50 anos, mas sua incidência também é presente em homens de diversas faixas etárias. Orientou-se sobre a importância da realização do autoexame das mamas para os homens e mulheres, embora seja uma doença incomum e represente apenas $1 \%$ de todos os cânceres de mama nos homens (SILVA, TOSCANI e GRAUDENZ, 2008). O projeto educativo em saúde buscou superar a perspectiva de transmissão de um conhecimento especializado utilizada majoritariamente nas atividades com a população.

As gestantes que aguardavam consulta para pré-natal e indivíduos que acompanhavam as crianças nas consultas de acompanhamento do crescimento e desenvolvimento infantil. Para estes grupos referiu-se sobre a importância da amamentação como forma de prevenção ao câncer de mama. De acordo com o ministério da saúde uma das vantagens da amamentação é a redução do risco de desenvolvimento do câncer de mama (BRASIL, 2001).

Para ambos os grupos foi discutido sobre a técnica correta de realização do autoexame das mamas, por este ser considerado método de maior acesso e baixo custo. Foram debatidas as reais diferenças entre autoexame e exame clínico, e esclarecido que apesar destes métodos serem reconhecidos como de menores sensibilidades, os estudos de imagem devem ser utilizados em grupos de maior risco e em situações de esclarecimentos de diagnóstico.

Contou-se com o conhecimento prévio da população presente e desta forma pôde-se esclarecer as principais dúvidas que os participantes referiram, dentre elas periodicidade e posicionamento para realização do autoexame, além da diferenciação 
Ensino, Saúde e Ambiente - V9 (1), pp. 33-42, Abril, 2016.

dos principais achados que estão dentro da normalidade ou não. $\mathrm{O}$ principal recurso usado neste momento foi um macromodelo de mama, além de fotografias que demonstravam a técnica correta.

Entende-se que os processos comunicativos que colocam em movimento os programas e projetos de educação em saúde é a permanência da ideia de que a "falta de saúde" é um fator que pode ser solucionado, individual ou coletivamente, desde que se disponha de informações técnico-científicas adequadas e/ou da vontade pessoal e política dos sujeitos expostos a determinados agravos à saúde (MEYER et al., 2006).

\section{(Re)orientação da solicitação de exames}

Analisou-se que na unidade de saúde não existiam parâmetros ou fluxos institucionalizados que direcionassem os profissionais para a distribuição equitativa das mamografias a serem ofertadas. O exame mamográfico não é realizado na USF, mas na atenção de média complexidade a partir da solicitação médica ou em mutirões realizados em meses específicos de campanha, como no caso do outubro rosa.

Com relação à elaboração do fluxograma para priorização das solicitações de exames mamográficos, utilizou-se os critérios estabelecidos pelo Ministério da Saúde em 2006, são eles: Mamografia para mulheres com idade entre 50 a 69 anos de idade, com intervalo máximo de 2 anos entre os exames, para mulheres a partir de 35 anos de idade, pertencentes a grupos populacionais com risco elevado de desenvolver câncer de mama, além de garantia de acesso ao diagnóstico, tratamento e seguimento para todas as mulheres com alterações nos exames realizados.

A importância da realização deste exame de forma criteriosa contribui para a efetivação de um rastreamento organizado, uma vez que quando este é ofertado para os grupos prioritários, pode reduzir em até $35 \%$ a mortalidade, desde que seja alcançada uma cobertura populacional igual ou superior a $70 \%$ da população-alvo (BRASIL, 2011).

\section{Educação em serviço}

Para a capacitação da equipe, foi realizada uma educação em serviço junto aos Agentes Comunitários de Saúde (ACS) e Técnicos de Enfermagem (TE). Objetivou-se dialogar com estes profissionais sobre a técnica correta de realização do autoexame das mamas. A proposta de conversa foi o modelo diametralmente oposto às "palestras", 
onde buscou-se fazer circular a palavra, valorizando as expressões, as experiências, as vivências, as representações sociais de cada trabalhador participante.

A proposta da realização dessa atividade estava a todo o momento direcionada a multiplicação do saber. Sendo o trabalho do agente comunitário, por exemplo, quase que predominantemente da realização de visitas, oportunizou-se para este grupo essa capacitação para que os ACS difundissem a informação apreendida dentro da comunidade em que atuam.

Com relação aos TE compreendeu-se que estes poderiam, após a habilitação para propagação da realização do autoexame, difundir o conhecimento dentro da unidade de saúde em que atuam durante a realização de diferentes procedimentos de enfermagem, principalmente direcionados a mulher.

\section{Dia de prevenção e combate ao câncer de mama}

Foi confeccionado convite personalizado a todas as mulheres pertencentes a área de adscrição da USF para o encerramento do "Outubro Rosa". A listagem dos nomes deu-se a partir de reunião na unidade de saúde com os ACS. Os ACS, elos entre comunidade-unidade-universidade, foram responsáveis pela entrega dos convites.

O dia do encerramento do projeto intervenção foi divulgado e intitulado por "Dia de prevenção e combate ao câncer de mama". Este dia ocorreu no último dia do mês de outubro. A atividade foi iniciada com a apresentação de todos organizadores do evento, enfermeirandas, docente, psicóloga nutricionista e fisioterapeuta e ACS. Participaram da atividade extensionista além destes, vinte e cinco mulheres da comunidade da área de abrangência da USF. As mulheres foram recepcionadas e presenteadas com um broche rosa cujo o formato de laço simboliza internacionalmente a luta contra o câncer de mama.

O primeiro momento foi conduzido pela psicóloga do NASF que discorreu sobre a necessidade do apoio a ser dispensado por aqueles que convivem com pessoas com câncer e que o individuo com câncer precisa expressar seus sentimentos por meio de conversas, desabafos, choros, apoio religioso e atividades que promovem prazer. A profissional ainda discutiu sobre quais são os sentimentos envolvidos no processo de convivência com o câncer de mama, por ser este o mais temido pelas mulheres devido à sua alta frequência e por este afetar a percepção de sexualidade uma vez que interfere, muitas vezes, na própria imagem pessoal da mulher. 


\section{Ensino, Saúde e Ambiente - V9 (1), pp. 33-42, Abril, 2016.}

Depois foi feita abordagem ilustrativa sobre a anatomia da mama e explicação prática sobre a técnica do auto-exame. Neste momento uma enfermeiranda discutiu junto com as participantes sobre a importância da realização mensal do autoexame das mamas e explicou que a técnica deve ser realizada após o período menstrual para mulheres que ainda menstruam e para as mulheres menopausadas deve ser escolhida uma data fixa no mês. Foram desmistificados os achados normais daqueles sugestivos de anormalidade, além disso as mulheres também foram orientadas quanto a real necessidade de realização de exames complementares a nível de investigação.

Todas as mulheres participaram ativamente, realizando conjuntamente o autoexame, o que propiciou troca de informação e possibilitou o esclarecimento das dúvidas quanto a técnica correta. Referenciou-se que a própria mulher que se autoexamina periodicamente é capaz de perceber alterações palpáveis em seu inicio o que diminui a chance da necessidade de retirada da mama no caso da confirmação do câncer.

No terceiro momento foi abordado por outra enfermeiranda os aspectos relevantes sobre a mama normal e a mama alterada através de uma ilustração contendo doze mamas com os diferentes sinais sugestivos de câncer: endurecimento, vermelhidão, alteração de veias, presença de buracos, secreção purulenta, protuberâncias, erosão de pele, reentrância de mamilo, assimetria mamária, nódulos e ardor. Salientou-se que os sinais sugestivos podem apresentar-se isoladamente ou associados.

Para avaliação do terceiro momento solicitou-se que as participantes se dividissem em cinco grupos e preenchessem conjuntamente um instrumento que continha fotografias de alguns sinais sugestivos para o câncer. Neste momento puderam visualizar os sinais e sugerir a nomeclatura destes, demonstrando assim que estavam aptas para reconhecê-los se por ventura viessem a aparecer em suas próprias mamas.

Discutiu-se no quarto momento, por outra enfermeiranda os exames complementares (ultrassonografia e mamografia), sendo explicado sobre quais as diferenças entre os exames, quais mulheres devem ter prioridade e sobre a importância do exame de ultrassom enquanto complementar ao exame de mamografia.

No quinto momento houve a participação da nutricionista que utilizou um jogo interativo com auxilio de um dado (com figuras de alimentos) como principal instrumento. As participantes deveriam jogar o dado e responder a pergunta sorteada no envelope correspondente ao número encontrado no dado. A proposta central dessa 
Ensino, Saúde e Ambiente - V9 (1), pp. 33-42, Abril, 2016.

discussão foi esclarecer os mitos existentes sobre alimentação adequada para indivíduos que convivem ou conviveram com o processo do câncer de mama.

No sexto momento, foi discutida pela fisioterapeuta a abordagem sobre aspectos relacionados à mastectomia e a reabilitação com exercícios simples, que podem ser realizados em domicílio. Logo após, procedeu-se ao encerramento da atividade.

\section{Considerações}

Buscou-se em todas as atividades desenvolvidas a participação das mulheres entendendo a importância de tornar o individuo parte do processo educativo, tornando assim a ação mais efetiva, pois o mesmo apreende as orientações teoricamente e tem a oportunidade de treinar/realizar o que foi proposto.

A universidade deve ser a instituição que se propõe a desenvolver com a comunidade, ações que se caracterizam como atividades de extensão. Essas ações são de natureza fundamental para que se estabeleça uma relação de parceria entre a universidade e a sociedade. $\mathrm{O}$ presente projeto proporcionou a vivência da troca de saberes entre acadêmicos, equipe de saúde da família e comunidade, ressignificando o processo de educação, baseado na construção compartilhada de conhecimento sobre o saber saúde, ampliando o espaço universitário e exercitando o compromisso social.

\section{REFERÊNCIAS:}

Associação Feminina de Educação e Combate ao Câncer (AFECC). Outubro Rosa Um toque pela vida. Disponível em http://www.afecc.org.br/.

BRASIL. Parto, aborto e puerpério: assistência humanizada à mulher. Secretaria de Políticas de Saúde, Área Técnica da Mulher. Brasília: Ministério da Saúde, 2001.

BRASIL. Ministério da Saúde. Secretaria de Gestão Estratégica e Participativa. Departamento de Apoio à Gestão Participativa e ao Controle Social. Saúde da mulher: um diálogo aberto e participativo. Brasília: Ministério da Saúde, 2010.

BRASIL. Controle do câncer de mama. Documento de consenso. Brasília: Ministério da Saúde, 2004

BRASIL. Secretaria de Atenção à Saúde. Departamento de Atenção Básica. Controle dos cânceres do colo do útero e da mama. Brasília: Ministério da Saúde, 2006.

FOGAÇA, E.I.C, GARROTE, L.F. Câncer de mama: atenção primária e detecção precoce. Arq Ciênc Saúde v. 11, n. 3, p. 179 181, 2004. 
Ensino, Saúde e Ambiente - V9 (1), pp. 33-42, Abril, 2016.

INSTITUTO NACIONAL DE CÂNCER. Rastreamento organizado do câncer de mama: a experiência de Curitiba e a parceria com o Instituto Nacional de Câncer. Rio de Janeiro: INCA; 2011

MAFRA, A.G.F.D. Zinco e câncer: uma revisão. Revista Saúde.com. v. 1, n. 2, p. 144 156, 2005.

MEYER, et al. "Você aprende. A gente ensina?" Interrogando relações entre educação e saúde desde a perspectiva da vulnerabilidade. Cad. Saúde Pública, Rio de Janeiro, v. 22 , n. 6, p. 1335-1342, 2006.

SILVA LM, TOSCANI V, GRAUDENZ MS. Câncer de mama masculino: uma doença diferente? Rev Bras Mastol.v. 18, n.4, p. 165 170, 2008.

SOUZA LRS, SOUZA NA, SOARES CJ, LINHARES EF, OLIVEIRA ZM, MEIRA LS. A mulher com câncer de mama e o convívio familiar. Revista Saúde.com. v. 9, p. 129 130, 2013. 